\title{
УСТОИЧИВОЕ РЕШЕНИЕ ЗАДАЧИ ОПТИМИЗАЦИИ ТОПЛИВНО-ЭНЕРГЕТИЧЕСКОГО БАЛАНСА (ТЭБ) РАЙОНА
}

G. RABKIN. RAJOONI KOTUSE- JA ENERGIABILANSI OPTIMEERIMISE OLESANDE POSIV LAHEND

G. RABKIN. ROBAST SOLUTION OF THE FUEL-POWER BALANCE OF AN ECONOMIC DISTRICT

(Представил И. Эпик)

В настоящее время оптимальное управление топливно-энергетическим комплексом осуществляется по математическим моделям энергетических хозяйств районов. Наибольшее распространение получила модель $\left[{ }^{1-3}\right]$, суть которой составляет распределительная задача линейного программирования

$$
\min \left(\sum_{i=1}^{m} \sum_{l=1}^{t} z_{i l} x_{i l}\right)
$$

при ограничениях

$$
\begin{array}{r}
\sum_{i=1}^{m} \eta_{i l} x_{i l} \geqslant Q_{l}, \quad l=\overline{1, t}, \\
\sum_{l=1}^{t} x_{i l} \leqslant x_{i}, \quad i=\overline{1, m},
\end{array}
$$

где $z_{i l}-$ стоимостный показатель $i$-го вида топлива у $l$-го потребителя, $\eta_{i l}-$ кпд $l$-го потребителя, $Q_{l}$ - полезная произведенная энергия $l$-го потребителя, $x_{i l}$ - искомый объем $i$-го топлива у $l$-го потребителя.

Оптимизация ТЭБ представляет собой нахождение решения ряда задач типа (1)-(3). Рассмотрим вопросы решения задач такого типа в условиях действия гипотезы: исходные данные $z_{i l}, \eta_{i l}, Q_{l}, x_{i}$ $(i=\overline{1, m} ; l=\overline{1, t})$ являются неопределенными и задаются интервалами изменения $\left[\bar{\varepsilon}_{(.)}, \tilde{\varepsilon}_{(.)}\right]$, где $(\cdot)$ обозначает соответствующий параметр исходных данных.

В дальнейшем будем придерживаться обозначений, введенных, в основном, в $\left[{ }^{4}\right]: \Omega_{\alpha}=\left[\overline{\varepsilon_{\alpha}}, \tilde{\varepsilon_{\alpha}}\right]-$ пространство реализаций неопределенного параметра (н. п.) $\alpha ; \varepsilon_{\alpha} \in \Omega_{\alpha}-$ ожидаемое значение н. п. $\alpha$; $\Delta_{\alpha}=\tilde{\varepsilon}_{\alpha}-\bar{\varepsilon}_{\alpha}-$ степень неопределенности; $k_{\alpha}=\left(\varepsilon_{\alpha}-\bar{\varepsilon}_{\alpha}\right) / \Delta_{\alpha}-$ қоэф. 
фициент относительного смещения; $I(\alpha)=I\left(\overline{\varepsilon_{\alpha}}, \varepsilon_{\alpha}, \tilde{\varepsilon}_{\alpha}\right)-$ общая характеристика н.п. $\alpha$. Очевидно, что последняя может быть представлена и в другой форме: $I(\alpha)=I\left(\varepsilon_{\alpha}, \Delta_{\alpha}, k_{\alpha}\right)$.

Рассмотрим задачу (1)-(3). В силу неопределенности исходных данных естественно предположить неопределенность решения данной задачи. Пусть общая характеристика решения $x_{i l}{ }^{0}$ задачи $(1)-(3)$ суть

$$
I\left(\underset{\varepsilon_{x i l}}{0} \Delta_{x i l}^{0}, k_{x i l}^{0}\right), \quad i=\overline{1, m}, \quad l=\overline{1, t}
$$

и пусть мы имеем правило выбора ожидаемых значений н. п. исходных данных. Введем следующие определения.

О п р еде лен и е 1. Ожидаемым значением н.n. $x_{i l}^{0}$ называется решение данной задачи при ожидаемых значениях н.п. исходных данных, T. е. задачи типа

$$
\left\{\min \sum_{i=1}^{m} \sum_{l=1}^{t} \varepsilon_{z i l} x_{i l} \mid \sum_{i=1}^{m} \varepsilon_{\eta i l} x_{i l} \geqslant \varepsilon_{Q l} ; \sum_{l=1}^{t} x_{i l} \leqslant \varepsilon_{x i} ; i=\overline{1, m}, \quad l=\overline{1, t}\right\} .
$$

Определение 2. Если в точке оптимального решения $x_{i l}{ }^{0}$ задачи (1)-(3) с общей характеристикой (4) имеют место соотношения

$$
\begin{aligned}
& \Omega \sum_{i \in I_{l}} \eta_{i l} x_{i l}^{0} \subseteq \Omega_{Q l}, \quad l=\overline{1, t}, \\
& \Omega \underset{l \in L_{l}}{\Sigma_{i l} x_{i l}^{0}} \subseteq \Omega_{x i}, \quad i \in I^{0},
\end{aligned}
$$

то такое решение называется решением, обеспечивающим устойчивость задаче (ОУЗ) (1)-(3) с неопределенными исходными данными.

Здесь $I_{l} \subset I=\{\overline{1, m}\}$ состоит из $m_{l}$ элементов таких, что для любого $i \in I_{l}$ решение $x_{i l}{ }^{0} \quad(l=\overline{1, t})$ входит в оптимальный план; $L_{i} \subset L=$ $=\{\overline{1, t}\}$ состоит из $t_{i}$ элементов таких, что для любого $l \in L_{i}$ решение $x_{i l}{ }^{0} \quad(i=\overline{1, m})$ входит в оптимальный план; $I^{0} \subset I-$ множество индексов $i$, для которых имеет место строгое равенство в (3).

Применительно к задаче оптимизации ТЭБ экономического района решение ОУЗ интерпретируется как решение, при котором любые колебания в поставках топлива потребителю в пределах пространства реализаций $\Omega_{x i l}{ }^{0}$ не приводят ни к дефициту топлива - (7), ни к перепроизводству или недопроизводству полезной энергии - (6) в рамках действия принятой гипотезы о характере исходной информации.

Оп реде лен ие 3 . Пусть I(.) - общиче характеристики н. п. исходных данных задачи (1)-(3), при которых существует решение ОУЗ. Тогда такой набор общих характеристик называется согласованным.

Имеет место следующая теорема, позволяющая при некоторых условиях находить решение задачи (1)-(3) в классе решений ОУЗ.

Т еорем а. Пусть выполнены следующие условия:

1) Н.n. исходных данных задачи (1)-(3) заданы согласно гипотезе;

2) имеет место одно из следующцх двух соотношений, определяющих согласованный выбор ожидаемых значений н.п. исходных данных

$$
\begin{array}{lll}
k_{Q l}=k_{\eta i l}=k_{x i}=0, & i=\overline{1, m}, & l=\overline{1, t}, \\
k_{Q l}=k_{\eta i l}=k_{x i}=1, & i=\overline{1, m}, \quad l=\overline{1, t} ;
\end{array}
$$


3) $\varepsilon_{x i l}{ }^{0}$ - ожидаемое решение задачи (1)-(3);

4) в точке $\varepsilon_{x i l}{ }^{0}$ выполнено соотношение

$$
d_{l}=\Delta_{Q l}-\sum_{i \in I_{l}} \varepsilon_{x i l}^{0} \Delta_{\eta i} \geqslant 0, \quad l=\overline{1, t} .
$$

Тогда, если

$$
\begin{aligned}
& \Delta_{x i l}^{0}=\min \left[d_{l} \varepsilon_{\eta i l} / \tilde{m}_{l} \tilde{\varepsilon}_{\eta i l} \bar{\varepsilon}_{\eta i l}, i \in I_{l}^{0}, l=\overline{1, t} ; \quad \Delta_{x i} / t_{i}, i \in I_{l}^{0}, l \in L_{i}\right], \\
& \Delta_{x i l}^{0}=d_{l} \varepsilon_{\eta i l} / m_{l} \tilde{\varepsilon}_{\eta i l} \bar{\varepsilon}_{\eta i l}, \quad i \in I_{l} \backslash I_{l}^{0}, \quad l=\overline{1, t}, \\
& k_{x i l}^{0}=k_{x i}, \quad i \in I_{l}, \quad l=\overline{1, t},
\end{aligned}
$$

то решение $x_{i i^{0}}$ задачи (1)-(3) с общей характеристикой $I\left(x_{i i}{ }^{0}\right)=$ $=I\left(\varepsilon_{x i l}{ }^{0}, \Delta_{x i l}{ }^{0}, k_{x i l}{ }^{0}\right)$ есть решение ОУЗ.

Пункты 1 и 2 теоремы определяют ожидаемые значения н. п. исходных данных, по которым находится ожидаемое решение $\varepsilon_{x i i^{0}}$ задачи (1) - (3) согласно определению 1. Отметим, что выбор одного из двух условий согласования осуществляется исходя из качественного анализа исходной информации и специфики решаемой задачи оптимизации ТЭБ.

Рассмотрим условие (10), выполнение которого необходимо для существования решения ОУЗ. В практических задачах оптимизации ТЭБ это условие обычно выполняется, ибо элементы матрицы технологических способов $\eta_{i l}-$ кпд установок, производящих необходимую энергию $Q_{l}$, оказываются в $2-4$ раза более устойчивыми, чем элементы вектора ограничений $Q_{l}\left[^{5,6}\right]$.

Задача оптимизации ТЭБ в данной постановке была реализована для нахождения оптимального состояния ТЭБ республик Прибалтики и Белоруссии.

\section{ЛИТЕРАТ У РА}

1. М а к а ров А. А., Методы исследования и оптимизации топливно-энергетического хозяйства, Автореф. докт. дис., Иркутск, 1969.

2. Макаров А. А,, Мелент ьев Л. А., Методы исследования и оптимизации энергетического хозяйства, Новосибирск, «Наука», 1973.

3. Методика оптимизации развития топливно-энергетического хозяйства (под ред. А. А. Макарова), Ч. I, Иркутск, 1966.

4. Н е с е ю к А. П., Автоматика, № 2, 55-63 (1979).

5. Б а р а б а н ер Х. 3., Теплоснабжение сельских населенных пунктов, Таллин, «Валгус», 1976.

6. В олконски й В. А., Принципы оптимального планирования, М., «Экономика», 1973.

Институт термофизики и электрофизики Академии наук Эстонской ССР

Поступила в редакцию 11/II 1981 